\section{Daily Spiritual Experiences: Examining Measurement Invariance by Gender in a Nationally Representative Sample}

Joe Chelladurai

\begin{abstract}
In this paper, I examine the Daily Spiritual Experience Scale (DSES) for measurement invariance across men and women. Data for this analysis is from the General Social Survey, a nationally representative dataset of participants from the United States. Using lavaan and semPlot packages in $\mathrm{R}, \mathrm{I}$ created nested models to test for levels of invariance. Results indicated that a brief 5-item DSES construct achieved scalar invariance across groups, suggesting that the construct holds true for both men and women. These findings add support for testing gender differences for DSES using the brief 5-item DSES construct.
\end{abstract}

Keywords: measurement invariance, religion, spirituality, latent variable modeling, lavaan

In recent research literature, it has well been established that women and men vary on their average levels of spirituality. This has been explained by various theories, although subjected to debate (Freese, 2004). For instance, women take less risks than men, or that women are more religious and spiritual as their socialization processes are different than men. And moreover, women have historically been engaged at home and as a result spend more time contemplating religious or spiritual things.

Regardless of theoretical explanations, women and men generally report different levels of spirituality. However, there is a need to first examine whether men and women think about religion and spirituality in the same way. This can be achieved using measurement equivalence testing which would help to then examine differences with more confidence.

\section{Method}

\section{Participants}

Participants for this study are from the 2004 General Social Survey. Conducted by the National Opinion Research Center, the GSS is a repeated cross-sectional survey with a nationally representative sample. Data was collected by interviewing in person and over telephone when in-person interviews were not possible. Topics of the survey included demographic characteristics, behavioral information, psychological and social constructs such as happiness, gender roles, financial choices, and political preferences.
Daily Spiritual Experiences Scale. The daily spiritual experiences scale was developed by Lynn Underwood to measure ordinary day to day spiritual experiences. The ten items included in the 2004 GSS survey were:

- I feel God's presence

- I am spiritually touched by the beauty of creation.

- I feel deep inner peace or harmony

- I ask for God's help in the midst of daily activities

- I feel guided by God in the midst of daily activities

- I desire to be closer to God or in union with Him

- I feel God's love for me, directly

- I feel God's love for me through others

- I find strength in my religion or spirituality

- I find comfort in my religion or spirituality

\section{Analysis Plan}

Based on Meredith's (1993) recommendations, I examined measurement invariance using a nested model.

\section{Results}

\section{Model Fit and Modification}

An overall model with ten items was created and checked if the model fit well with the data. Model fit was poor when all items were used. To improve model fit, modification indices were requested, and appropriate variables were removed. Some of the items that were removed were comparable to items that were in the final set of items. For example, "I find strength in my religion or spirituality" was similar to "I find comfort in my religion or spirituality." As a result of this process, five items that resulted with good model fit were selected $(C F I=998, T L I=$ 996, $\mathrm{RMSEA}=0.039, \mathrm{SRMR}=0.008$.

The figure below represents the measurement model of the five items of the daily spiritual experiences scale.

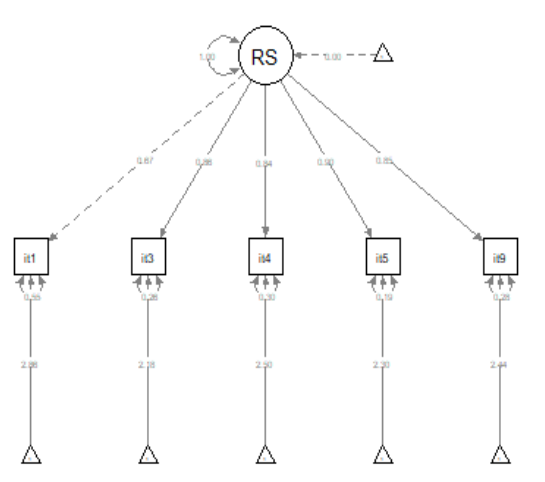




\section{Measurement Model by Group}

Next, I created a factor variable to examine the measurement model according to gender. Then, I examined the fit indices of the model with only men, and the model with only women. Both had good model fit, with women (CFI $=.999, \mathrm{TLI}=.999, \mathrm{RMSEA}=.018, \mathrm{SRMR}=.008)$ having $\mathrm{a}$ slightly better model fit than men $(C F I=.996, T L I=.991$, RMSEA $=.057$, SRMR $=.012$ ).

\section{Configural Invariance}

I then examined nested models to see what level of invariance this measurement model reached. First, I examined the configural invariance model, where all parameters were allowed to vary. This model indicated good model fit (CFI = .998, TLI .995, RMSEA $=.041$, SRMR $=.01)$ and provided evidence for configural invariance.

\section{Metric Invariance}

Next, I examined whether the model achieved metric invariance. This is done by constraining the item loadings to be equal across groups. Results from the metric model indicated good model fit $(C F I=.997, \mathrm{TLI}=.996$, RMSEA $=.037, \mathrm{SRMR}=.022$ ). This suggested that the model was invariant across groups at the level of the factor loadings.

\section{Scalar Invariance}

To establish whether the measurement model had strong invariance, the intercepts were constrained along with the loadings. Results from the scalar model indicated good model fit (CFI $=.004, \mathrm{TLI}=.993, \mathrm{RMSEA}=.049, \mathrm{SRMR}=.027)$. This suggested that the model was invariant across groups at the level of factor loadings and the intercepts.

\section{Strict Invariance}

As an added analytic procedure, the model was tested whether there was strict invariance. To conduct strict invariance, the variances of the observed items were constrained in addition to the intercepts and the factor loadings. Results from the strict model indicated good model fit $(\mathrm{CFI}=.992, \mathrm{TLI}=.993, \mathrm{RMSEA}=.048, \mathrm{SRMR}=.032)$. This indicated that strict invariance was reached. The model fit indices of all models are presented below:

\begin{tabular}{lllllll}
\hline Model & CFI & TLI & RMSEA & SRMR & Chi Square & df \\
\hline Configural & .998 & .995 & .041 & .010 & 20.74 & 10 \\
Metric & .997 & .996 & .037 & .022 & 26.187 & 14 \\
Scalar & .994 & .993 & .049 & .027 & 45.906 & 18 \\
Strict & .992 & .993 & .048 & .032 & 56.5 & 23 \\
\hline
\end{tabular}

\section{Discussion}

Results from the analysis indicate that the reduced 5-item DSES measure was invariant across groups. Measurement invariance was achieved at the strict level when variances, intercepts, and loadings were constrained.

The final model supports further examination of group differences in future research.

Findings should be taken with caution, however. The major drawback of the study is that best fitting model resulted with only five items when compared with the original ten items. This has severe restrictions as to which items can be used to measure and compare the levels of daily spiritual experiences.

Measuring spirituality has other challenges as there is seldom one defining type of spirituality across a wide range of participants such as a nationally representative sample. It is therefore important for future studies to focus exclusively on spirituality to examine what type of religious and spiritual beliefs and observances are pertinent to the individual. Global measures such as religious affiliation and participation may provide some indication, but with current trends indicating an increase in spirituality and a decline in religiosity (Pew Research Center, 2019) there is a need for more measures such as the Daily Spiritual Experiences Scale.

\section{References}

Freese, J. (2004). Risk preferences and gender differences in religiousness: Evidence from the World Values Survey. Review of Religious Research, 88-91.

Pew Research Center (2019). In U.S., decline of Christianity continues at rapid pace. https://www.pewforum.org/2019/10/17/in-u-s-decline-of-christianitycontinues-at-rapid-pace/

Meredith, W. (1993). Measurement invariance, factor analysis and factorial invariance. Psychometrika, 58(4), 525-543.

Appendix

Syntax

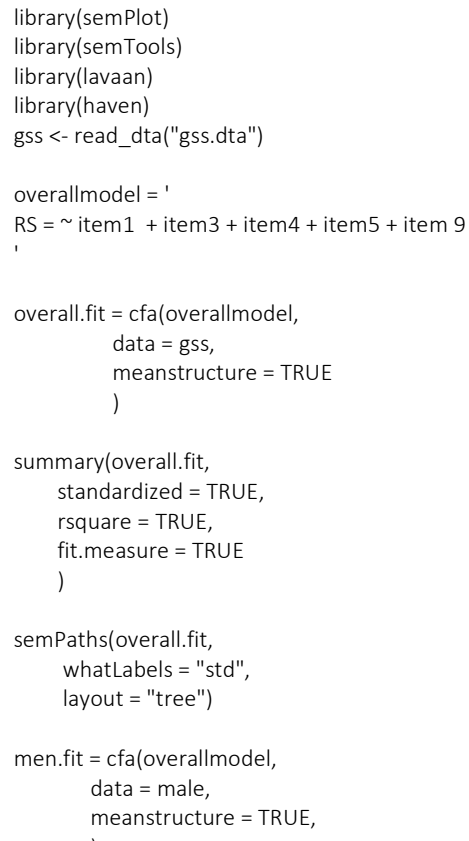


summary(men.fit,

standardized $=$ TRUE

rsquare $=$ TRUE

fit.measure $=$ TRUE)

women.fit = cfaloverallmodel, data $=$ female, meanstructure $=$ TRUE)

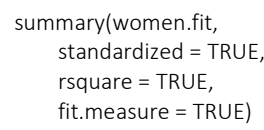

cfa <- cfa(overallmodel, gss, meanstructure = TRUE, group = "sex") summary(cfa)

\section{cfa1 <- summary(cfa, \\ standardized $=$ TRUE, \\ rsquare $=$ TRUE \\ fit. measure $=$ TRUE)}

metric <- cfa(overallmodel, gss, meanstructure = TRUE, group = "sex", group.equal= c("loadings"))

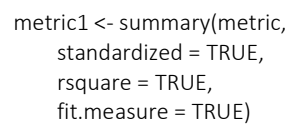

scalar <- cfa(overallmodel, gss, meanstructure = TRUE, group = "sex", group.equal= c("loadings", "intercepts"))

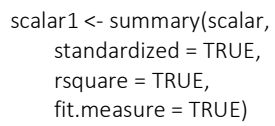

strict $<-$ cfa(overallmodel, gss, meanstructure = TRUE, group = "sex", group.equal= c("loadings", "intercepts", "residuals"))
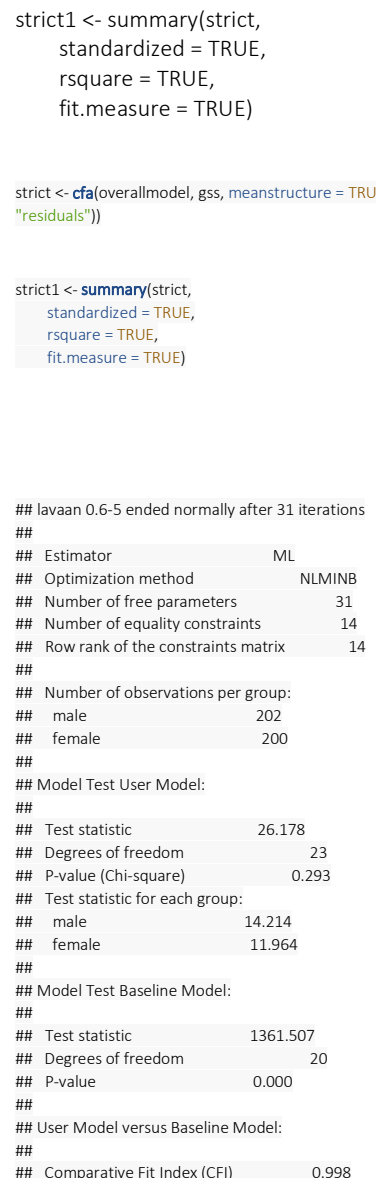

\#\# Comparative Fit Index (CFI)
\#\# Tucker-Lewis Index (TLI) 0.998

\#\#

\#\# Loglikelihood user model (HO) $\quad-3107.121$

\#\# Loglikelihood unrestricted model (H1) $\quad-3094.032$

\#\# Akaike (AIC) $\quad 6248.242$

\#\# Bavesian (BIC) 6316181

\#\# Sample-size adjusted Bayesian (BIC) $\quad 6262.239$

\#\#

\#\# Root Mean Square Error of Approximation:

\# RMSEA 0.026

\#\# 90 Percent confidence interval - lower $\quad 0.000$

\#\# 90 Percent confidence interval - upper 0.066

\#\# Standardized Root Mean Square Residual:

\#\# SRMR 0.031

\#\#

\#\# Parameter Estimates:

\#\# Information Expected

\# Information saturated (h1) model Structured

\# Standard errors Standard

\#\#

\#\# Group 1 [male]:

\#\# Latent Variables:

\#\# Estimate Std.Err $z$-value $P(>|z|)$ Std.lv Std.al

\#\# $\mathrm{RS}=\sim$

$\begin{array}{lllll}\text { \#\# } & \text { item1 } & 1.000 & 0.958 \quad 0.662\end{array}$

$\begin{array}{lllllllll}\text { \#\# } & \text { item3 } & \text { (.p2.) } & 1.511 & 0.099 & 15.223 & 0.000 & 1.448 & 0.855\end{array}$

$\begin{array}{lllllllll}\text { \#\# } & \text { item4 (.p3.) } & 1.357 & 0.091 & 14.876 & 0.000 & 1.300 & 0.830\end{array}$

$\begin{array}{lllllllll}\text { \# } & \text { item5 (.p4.) } & 1.531 & 0.097 & 15.732 & 0.000 & 1.466 & 0.893\end{array}$

$\begin{array}{lllllllll}\text { \# } & \text { item9 (.p5.) } & 1.396 & 0.093 & 15.074 & 0.000 & 1.337 & 0.844\end{array}$

\#\# Estimate Std.Err $z$-value $P(>|z|)$ Std.lv Std all

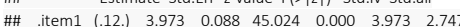

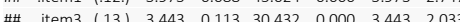

$\begin{array}{lllllllll}\text { \#\# } & .1 t e m 3 & (13 .) & 3.443 & 0.113 & 30.432 & 0.000 & 3.443 & 2.033\end{array}$

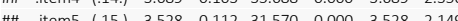

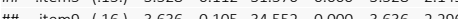

$\begin{array}{llllllllllllllll}\text { \# } & \text { \#.teng (16.) } & 3.636 & 0.105 & 34.552 & 0.000 & 3.528 & 2.296\end{array}$

\#\# Variances:

\#\# Estimate Std. Err $z$-value $P(>|z|)$ Std.lv Std.all

$\begin{array}{llllllllll}\text { \# } & \text { item1 (.p6.) } & 1.174 & 0.089 & 13.257 & 0.000 & 1.174 & 0.561\end{array}$

$\begin{array}{llllllllll}\text { \#\# } & \text { item3 (.p7.) } & 0.772 & 0.071 & 10.885 & 0.000 & 0.772 & 0.269\end{array}$

$\begin{array}{lllllllll}\text { \#\# } & \text { item4 (.p8.) } & 0.761 & 0.066 & 11.501 & 0.000 & 0.761 & 0.310\end{array}$

$\begin{array}{llllllllll}\text { \#\# } & \text { item5 } & \text { (.p9.) } & 0.546 & 0.058 & 9.397 & 0.000 & 0.546 & 0.202\end{array}$

$\begin{array}{lllllllll}\text { \#\# } & \text {.item9 } & \text { (.10.) } & 0.720 & 0.064 & 11.176 & 0.000 & 0.720 & 0.287\end{array}$

$\begin{array}{llllllllll}\text { \#\# } & \text { RS } & 0.918 & 0.142 & 6.452 & 0.000 & 1.000 & 1.000\end{array}$

\#\# R-Square:

\#\# Estimate

H

\#

$\begin{array}{lll}\# \# & \text { item4 } & 0.690 \\ \text { \# } & \text { item5 } & 0.798\end{array}$

\#\# $\quad$ item9 $\quad 0.713$

\#\#

\#\# Group 2 [female]

\#\# Latent Variables:

\#\# Estimate Std.Err $z$-value $\mathrm{P}(>|z|)$ Std.lv Std.al

\#\#S $=\sim$

$\begin{array}{lllll}\text { \# } & \text { item1 } & 1.000 & 0.956 & 0.662\end{array}$

$\begin{array}{llllllllll}\text { \# } & \text { item3 (.p2.) } & 1.511 & 0.099 & 15.223 & 0.000 & 1.444 & 0.854\end{array}$

$\begin{array}{lllllllll}\text { \# } & \text { item4 (.p3.) } & 1.357 & 0.091 & 14.876 & 0.000 & 1.297 & 0.830\end{array}$

$\begin{array}{lllllllll}\text { \# } & \text { item5 (.p4.) } & 1.531 & 0.097 & 15.732 & 0.000 & 1.463 & 0.893\end{array}$

\#\# $\quad$ item9 (.p5.) $1.396 \quad 0.093 \quad 15.074$

\#\# Intercepts:

\#\# Estimate Std.Err $z$-value $P(>|z|)$ Std.lv Std.all

$\begin{array}{lllllllll}\text { \# } & \text {.item1 } & \text { (.12.) } & 3.973 & 0.088 & 45.024 & 0.000 & 3.973 & 2.749\end{array}$

$\begin{array}{lllllllll}\text { \# } & \text { item3 } & (.13 .) & 3.443 & 0.113 & 30.432 & 0.000 & 3.443 & 2.036\end{array}$

$\begin{array}{llllllllll}\text { \# } & \text { item4 (.14.) } & 3.689 & 0.103 & 35.688 & 0.000 & 3.689 & 2.360\end{array}$

$\begin{array}{llllllllll}\text { \# } & \text { item5 } & (.15 .) & 3.528 & 0.112 & 31.570 & 0.000 & 3.528 & 2.152\end{array}$

$\begin{array}{lllllllll}\text { \#\# } & \text {.item9 (.16.) } & 3.636 & 0.105 & 34.552 & 0.000 & 3.636 & 2.300\end{array}$

$\begin{array}{lllllllll}\# \text { \#\# } & \text { RS } & 0.420 & 0.102 & 4.118 & 0.000 & 0.440 & 0.440\end{array}$

(1)

\#\# Variances:

\#\# Estimate Std.Err $z$-value $P(>|z|)$ Std.lv Std.al

$\begin{array}{lllllllll}\text { \#\# } & \text { item1 (.p6.) } & 1.174 & 0.089 & 13.257 & 0.000 & 1.174 & 0.562\end{array}$

$\begin{array}{lllllllll}\text { \#\# } & \text {.item3 } & \text { (.p7.) } & 0.772 & 0.071 & 10.885 & 0.000 & 0.772 & 0.270\end{array}$

\#\# $\quad$.item4 (.p8.) $0.761 \quad 0.066 \quad 11.501 \quad 0.0000 .761 \quad 0.311$

$\begin{array}{lllllllll}\text { \#\# } & \text {.item5 } & \text { (.p9.) } & 0.546 & 0.058 & 9.397 & 0.000 & 0.546 & 0.203\end{array}$

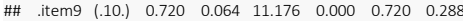

$\begin{array}{llllllllll}\text { \#\# } & \text { RS } & 0.914 & 0.142 & 6.435 & 0.000 & 1.000 & 1.000\end{array}$

\#\# R-Square:

\#\# Rstimate

\# item $1 \quad 0.438$

item $3 \quad 0.730$

\#\#

$\begin{array}{lll}\text { \#\# } & \text { item5 } & 0.797 \\ \text { \# } & \text { item } 9 & 0.712\end{array}$ 